\title{
Parallel Genetic Algorithm for Channel Routing
}

\author{
B. B. Prahlada Rao, L. M. Patnaik ${ }^{\dagger}$ and R. C. Hansdah ${ }^{\ddagger}$ \\ Indian Institute of Science, Bangalore - 560 012, India
}

\begin{abstract}
In this paper, we propose a new channel routing algorithm based on genetic approach. This involves designing a new encoding scheme and an evaluation function used by the genetic algorithm(GA) for channel routing problem. The algorithm has been implemented as both sequential and distributed GA. The speedup achieved is positive and encouraging.
\end{abstract}

\section{Introduction}

In the physical design of VLSI circuits, a significant portion (approximately $65-80 \%$ of total chip area) is used for interconnections. Efficient routing of these interconnections reduces chip size. Hence, routing has received a great deal of attention from the researchers. The concept of channel routing was first proposed by Hashimoto[1] in 1971. Since then various algorithms have been proposed for channel routing in the past two decades[2, 4]. The objective of channel router is to interconnect terminals sitting on the opposite sides of a rectangular region called channel, using a minimum number of tracks. This is an NP-complete problem[3] . Most of the proposed channel routing algorithms $[2,4]$ are sequential in nature, i.e., they work on one possible routing solution at a time. The motivation for our channel routing strategy is to develop a parallel channel router using genetic approach. This methodology starts with a set of initial solutions, and every time it tries to combine two good solutions to produce another solution which inherits the features of the parents, thereby trying to search for a new potential solution.

Genetic algorithms(GA) are robust stochastic search algorithms based on biological evolution models. The basic concepts of GA were developed by Holland in 1975[5]. The algorithm operates on a population of individuals which represent points in the search space. Each individual has some fitness value measured by

\footnotetext{
"Computer Science and Automation Department

$\dagger^{\dagger}$ Microprocessor Applications Laboratory

$\ddagger_{\text {Computer Science and Automation Department }}$
}

an evaluation function. The time steps for evolution in the GA are called generations. In each generation, the algorithm selects the fittest individuals and reproduces the offsprings for the next generation using crossover and mutation operators. The traditional GA is described in [4]. A GA to solve a problem must have the following components: (i) A genetic encoding scheme to represent the solution space of the problem. (ii) An evaluation function that evaluates to show how well/bad the solutions are satisfying the constraints of the problem. (iii) Selection of the parameter values used in GA(POPSIZE, MAXGEN, probabilities of applying the genetic operators, ..etc). (iv) A set of genetic operators, viz., selection, crossover, and mutation and a few more, have to be defined to suit the application.

\section{Channel Routing Problem}

A horizontal channel is assumed. The routing is done in two layers. A net is a set of terminals to be interconnected and is denoted by $n_{i}$. Let 1 be the length of the channel and $N$ be the set of nets to be routed. The set of terminals on the top(bottom) of the channel are denoted by $T(B)$.

$$
\begin{aligned}
& \text { Let } \\
& T=\left\{t_{1}, t_{2}, \ldots, t_{l}\right\}, \\
& B=\left\{b_{1}, b_{2}, \ldots, b_{1}\right\} \text {, } \\
& N=\left\{n_{1}, n_{2}, \ldots, n_{k}\right\}, n_{i} \subset T \bigcup B ;
\end{aligned}
$$$$
\text { where }
$$

$t_{i}$ or $b_{i}$ can take value $0, \mathrm{~L}, \mathrm{R}$ or any value from $N$;

0 represents that the terminal is not connected to any net;

$L$ represents that the terminal is connected to a net entering from the left side of the channel;

$R$ represents that the terminal is connected to a net entering from the right side of the channel.

The objective of CHR is to assign tracks in the channel to the given set of nets using minimum number of tracks and satisfying the following constraints.

. No horizontal constraints is violated.

. No vertical constraints is violated. 

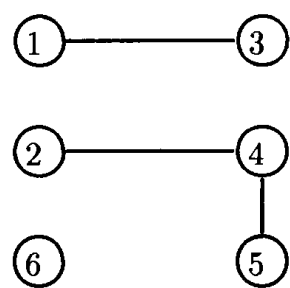

Figure 1: An HNCG Graph

Horizontal constraints can be represented using the horizontal non_constraint graph(HNCG) [4], $\mathrm{HNCG}=(\mathrm{N}, \mathrm{E})$, where $\mathrm{N}$ is the set of nets, and an undirected edge $\left(n_{i}, n_{j}\right) \in \mathrm{E}$ indicates that the horizontal spans of nets $n_{i}, n_{j}$ do not overlap.

\section{Problem Formulation}

The channel routing problem is to find a partitioning of the HNCG graph with minimum number of disjoint clusters(complete subgraphs) such that all the nets in a cluster can be routed in a track of the channel.

\subsection{Encoding Scheme, Crossover, Mu- tation and Evaluation Function for Channel Routing Problem}

We encode a $\mathrm{k}$-node HNCG graph using $\mathrm{k}(\mathrm{k}-1) / 2$ binary bits. The encoding scheme is based on our observation that the adjacency matrix representation of a graph is always symmetric. Hence, the lower triangle of the adjacency matrix is represented as a linear bit string. In our encoding scheme, each connected subgraph is interpretated as complete subgraph. Hence, the HNCG graph of fig. 1 is encoded as 010010010100000 .

Single point crossover [6] has been implemented in our experimentation. The mutation operator arbitrarily alters one or more bits in a string. We have proposed a new evaluation function for channel routing problem as shown below.

$$
f(w, U, H, V)=\frac{K_{1}}{w^{2}+\lambda_{1} U+\lambda_{2} H+\lambda_{3} V}
$$

where

$w=$ Number of tracks (width) of the routing

$H=$ Total number of horizontal constraint violations

$V=$ Total number of vertical constraint violations

$U=$ Measure of the sparcity of the tracks in a routing solution

$K_{1}, \lambda_{1}, \lambda_{2}$ and $\lambda_{3}$ are constants.

\section{Implementation and Analysis of Re- sults}

For our implementation, we used CDAC's PARAM machine which is a 64 node MIMD machine. The PE's can be reconfigured. We implemented the parallel genetic algorithm model of $[7,8]$. We observed a speedup of 2 to 3 (depending on the topology used) for 8 Processing Elements. This low speed up is because of the large communication overhead due to the total broadcast of the migrants to all PE's. Experimentation to reduce the communication overhead and increase the speedup is in progress. The results are positive and encouraging.

\section{References}

[1] A.Hashimoto and J.Stevens. Wire Routing by Optimizing Channel Assignment Within Large Apertures, Proceedings of 8th ACM/IEEE Design Automation Conference, 1971, pp. 214-224.

[2] H.W.Leong, D.F.Wong and C.L.Liu. A Simulated Annealing Channel Router, Proceedings of IEEE Intl. conf. on CAD (ICCAD) 1985, pp. 226-228.

[3] T.G.Szymanski. Dogleg Channel Routing is NPComplete, IEEE Tr. on CAD, Vol. CAD-4, No-1, pp. 31-41, January 1985.

[4] R.K.Pal, A.K.Dutta, S.P.Pal and A.Pal. Resolving Horizontal Constraints and Minimizing Net Wire Length for VHV Channel Routing, IIT(KGP) Tech. report no: TR/IIT/CSE/92/01, Sept 18 1992.

[5] John Holland. Seminal Work, Adaptation in Natural and Artificial Systems, Ph.D. Thesis, MIT 1975.

[6] David E.Goldberg. Genetic Algorithms in Search, Optimization and Machine Learning. Addison Wesley Publishing Co. Inc. 1989.

[7] R. Tenese. Distributed Genetic Algorithms for Function Optimization, Univ. of Michigan, $\mathrm{Ph} . \mathrm{D}$ thesis 1989.

[8] J.P.Cohoon et al. Distributed Genetic Algorithms for the Floorplan Design Problem, IEEE Tr. on CAD, Vol.10, April 1991, pp. 484-492. 